\title{
Evaluation of Relationship Between Tp-E Interval Values and QT Dispersion in Electrocardiogram in Children With B12 Vitamin Deficiency
}

\author{
- Nurcan Solak, • Canan Yolcu, • Murat Elevli, • Kamil Sahin \\ University of Health Sciences Turkey, Haseki Training and Research Hospital, Department of Pediatrics, Istanbul, Turkey
}

\section{Abstract}

\begin{abstract}
Aim: B12 vitamin deficiency may cause cardiac autonomic dysfunction, heart rate variability, endothelial dysfunction and subclinical atherosclerosis, a significant degree of reduction in heart ejection fraction and deformation of the left ventricular myocardium. We aimed to investigate the effect of B12 deficiency in children on cardiac electrical activity by comparing the Tp-e and QT dispersion values between healthy children and children with B12 deficiency.
\end{abstract}

Methods: The demographic data, laboratory results electrocardiography and echocardiography results of isolated B12 patients ( $\mathrm{n}=64$ ) with vitamin B12 deficiency and control group ( $n=64)$ included in the study between 15.3.2020 and 15.06.2020 were prospectively recorded and comparatively analyzed.

Results: A total of 128 patients included in the study. Corrected QT (QTc) value and Tp-e interval, were statistically significantly higher in the patient group $(\mathrm{p}<0.05)$. Tp-e/QT and Tp-e/QTc ratios in the patient groups were statistically significantly higher than the ones in the control group $(p<0.05)$.

Conclusion: Repolarization differences in B12 deficient children when compared to those of healthy children, might reflect subclinical symptoms.

Keywords: Child, vitamin B12 deficiency, QT dispersion, repolarization, Tp-e interval

\section{Introduction}

B12, a water-soluble vitamin, acts as a coenzyme in the synthesis of DNA required for cell division and proliferation, myelinization of neuronal cells and in the metabolisms of lipids and carbohydrates. By affecting the activity of the sympathetic and parasympathetic system, vitamin B12 deficiency causes heart rate variability and cardiac autonomic dysfunction and also results in endothelial dysfunction and subclinical atherosclerosis with its role in cellular metabolic pathways inhibiting homocysteine metabolism, lipid peroxidation and free radical formation. There are studies where vitamin B12 deficiency is associated with left ventricular myocardial deformation $(1,2)$.

Left ventricular dysfunction plays a key role in the development of arrhythmias. Prolonged QT interval increases the sensitivity of the heart to ventricular arrhythmias. The QT dispersion and Tp-e interval values are recognized as an indicator for ventricular repolarization. $(3,4)$. Prolonged QT dispersion and Tp-e interval values indicate a predisposition to ventricular arrhythmias.

In the literature, several studies show that vitamin B12 deficiency causes cardiac autonomic dysfunction and subclinical atherosclerosis in the pediatric age group $(1,5)$. Autonomic dysfunction caused by vitamin B12 deficiency and cardiac dysfunction secondary to anemia, its role in pathways preventing the formation of free radicals, its effect on endothelial reconstruction and the associated cardiac complications may make the heart susceptible to arrhythmias (6-8).

In our study, we aimed to investigate the effect of vitamin B12 deficiency on cardiac electrical activity in children by comparing Tp-e interval and QT dispersion values of children with isolated vitamin B12 deficiency to Tp-e interval and QT dispersion values of healthy children.

Address for Correspondence: Kamil Sahin, University of Health Sciences Turkey, Haseki Training and Research Hospital, Department of Pediatrics, Istanbul, Turkey Phone: +90 5324059706 E-mail: drkamil_sahin@hotmail.com ORCID: orcid.org/0000-0002-0443-2148 Received: 04.02.2021 Accepted: 28.03.2021 


\section{Methods}

\section{Study Design}

Ethical committee approval was obtained for our study from the Istanbul Training and Research Hospital, Clinical Research Ethics Committee of our hospital with the decision numbered 2020-06 and dated 11/03/2020. The families were informed about the study and their written consents were obtained.

Patients only with vitamin B12 deficiency were included in the study from among children who do not have any chronic disease (hypertension, diabetes mellitus, congenital or acquired heart disease, thyroid disease, chronic lung, liver and kidney disease, etc.) and whose other vitamin values (vitamin $D$, folate and ferritin) are in the normal range. The control group comprised of patients whose all vitamin values, including vitamin B12, were normal, and who did not have any additional chronic diseases and applied to the Pediatric Cardiology Outpatient Clinic for a routine pediatric examination or report for making sports and were not found to suffer from any problem and did not take any medication.

The demographic data of the patients were obtained from the patient files and the hospital information management system. Physical examinations, blood tests (hemogram, glucose, urea, creatinine, upper, lower, electrolytes, vitamin B12, folate, $25-\mathrm{OH}$ vitamin $\mathrm{D}$, ferritin, iron, iron-binding capacity, T4 TSH), age, gender, electrocardiography (ECG) and echocardiography (ECO) findings of the patients (taken prior to the initiation of the vitamin B12 deficiency treatment) between 15.3.2020 and 15.06.2020 were recorded prospectively in our clinical data registry form.

\section{Electrocardiogram Assessments}

Their ECGs were assessed by the same pediatric cardiologist. ECGs were taken by a 12-lead device (Schiller Cardiovit At-102 plus) with velocity $25 \mathrm{~mm} / \mathrm{sec}$ and amplitude $10 \mathrm{~mm} / \mathrm{mV}$. A magnifying glass and a manual ruler were used during the examination of ECG samples in order to increase the sensitivity of P wave, PR interval, QRS complex, QT interval, Corrected QT (QTc), Tp-e, Tp-e/QT, Tp-e/QTc, QT dispersion, and QTc dispersion calculations. The waves and intervals measured in our study are shown in Figure 1.

Echocardiographic examinations were performed with 2-dimensional and color Doppler echocardiography device (GE Vivid S5®). Echocardiographic assessment of all cases included in the study was performed by the same experienced pediatric cardiologist. In all cases, classical echocardiographic measurements, i.e., left ventricular enddiastolic diameter (LVEDD), left ventricular end-systolic diameter (LVESD), end-diastolic interventricular septal diameter (IVSd), end-diastolic left ventricular posterior wall

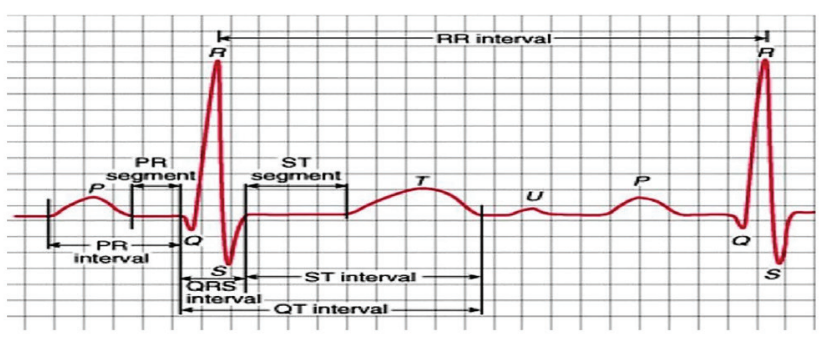

Figure 1: Waves and intervals making up the ECG ECG: Electrocardiography

diameter (LVPWd), ejection fraction (EF) and shortening fraction (SF) measurements were made.

\section{Statistical Analysis}

SPSS 15.0 for Windows program was used for statistical analysis. Descriptive statistics: Categorical variables are expressed in numbers and percentages and numerical variables are expressed in mean, standard deviation, minimum and maximum values. When the numerical variables met the normal distribution condition, the Student's t-test was used for the comparison of two independent groups, and the Mann-Whitney $U$ test was used when the normal distribution condition was not met. In independent groups, the rates were compared by chisquare test. The relationships between numerical variables were analyzed with Pearson's correlation analysis when the normal distribution condition was met, and when the normal distribution condition was not met, Spearman correlation analysis was used. The significance level alpha was accepted $p<0.05$.

\section{Results}

A total of 128 patients (64 patients and 64 controls) were included in the study. Of the 64 patients included in the study, 33 (51.6\%) were female and $31(48.4 \%)$ were male. The control group consisted of 33 (53.9\%) girls and $31(48.4 \%)$ boys. No statistically significant difference was found between the two groups in terms of gender distribution $(p=1)$. The mean age of the patient group was $11 \pm 2.7$ years and the mean age of the control group was $10.8 \pm 2.3$ years. No statistically significant difference was found between the patient and control groups in terms of the mean age $(p=0.428)$.

In the patient group, the mean vitamin B12 level was $144 \pm 30.3$, whereas in the control group, the mean vitamin B12 level was $310.6 \pm 131.9$. Folate level was found to be statistically significantly lower in the patient group compared to the control group $(p=0.035)$. However, it remained in the range of normal values in both groups (Table 1).

When all cases were evaluated, a weak positive correlation was found between vitamin B12 level and white 
Table 1. The distribution of vitamin and iron variables of the patient and control groups

\begin{tabular}{|c|c|c|c|c|c|}
\hline & \multicolumn{2}{|c|}{ B12 deficiency group } & \multicolumn{2}{|c|}{ Control group } & \multirow[b]{2}{*}{$p$} \\
\hline & Mean \pm SD & Minimum to maximum & Mean \pm SD & Minimum to maximum & \\
\hline Vitamin B12 (180-914 (pg/mL) & $144.0 \pm 30.3$ & $50-191$ & $310.6 \pm 132$ & $200-862$ & $<0.001^{\mathrm{a}}$ \\
\hline Folate $(5.9-24 \mathrm{ng} / \mathrm{mL})$ & $10.0 \pm 2.8$ & $4.69-17.68$ & $11.1 \pm 2.9$ & $5.21-18.2$ & $0.035^{b}$ \\
\hline Ferritin $(11-316 \mathrm{ng} / \mathrm{mL})$ & $23.5 \pm 7.4$ & $12-49.6$ & $27.3 \pm 13.1$ & $11.7-79.6$ & 0.179 \\
\hline TIBC1 (250-450 mcg/dL) & $285.8 \pm 49.1$ & $210-418$ & $283.3 \pm 49.2$ & $172-433$ & 0.778 \\
\hline Iron $(60-180 \mathrm{mcg} / \mathrm{dL})$ & $78.1 \pm 26.8$ & $23-170$ & $79.1 \pm 26.3$ & $37-160$ & 0.832 \\
\hline $25-\mathrm{OH}$ Vitamin D $(14-50 \mathrm{ng} / \mathrm{mL})$ & $17.1 \pm 3.7$ & $12-37.5$ & $18.5 \pm 4.3$ & $13.5-33.8$ & 0.079 \\
\hline
\end{tabular}

Table 2. Electrocardiographic findings of the patient and control groups

\begin{tabular}{|c|c|c|c|c|c|}
\hline & \multicolumn{2}{|c|}{ B12 group deficiency } & \multicolumn{2}{|c|}{ Control group } & \multirow[b]{2}{*}{$p$} \\
\hline & Mean \pm SD & \begin{tabular}{|l|}
$\begin{array}{l}\text { Minimum to } \\
\text { maximum }\end{array}$ \\
\end{tabular} & Mean \pm SD & \begin{tabular}{|l|} 
Minimum to \\
maximum
\end{tabular} & \\
\hline Heart rate (minute) & $83.8 \pm 14.8$ & $54-135$ & $88.1 \pm 16.0$ & $59 / 127$ & 0.118 \\
\hline$P(\sec )^{1}$ & $0.09 \pm 0.01$ & $0.07-0.12$ & $0.09 \pm 0.01$ & $0.06-0.12$ & 0.835 \\
\hline $\mathrm{PR}(\mathrm{sec})^{1}$ & $0.12 \pm 0.01$ & $0.09-0.15$ & $0.12 \pm 0.02$ & $0.09-0.16$ & 0.977 \\
\hline QRS $(\mathrm{sec})^{1}$ & $0.08 \pm 0.01$ & $0.07-0.10$ & $0.08 \pm 0.01$ & $0.07-0.11$ & 0.877 \\
\hline $\mathrm{QT}(\mathrm{msec})^{2}$ & $327.9 \pm 23.2$ & 273-396 & $328.3 \pm 44.7$ & $33-388$ & 0.164 \\
\hline QTc $(\mathrm{msec})^{2}$ & $393.5 \pm 18.8$ & $335-430$ & $405.5 \pm 18.7$ & $360-445$ & $<0.001^{a}$ \\
\hline
\end{tabular}

blood cell (WBC) and platelet values $(r=0.044 ; r=0.016$, respectively). No statistically significant relationship was found between other hemogram parameters and vitamin B12 level ( $p>0.05)$. No statistically significant difference was found in laboratory parameters in both groups $(p>0.05)$.

QTC was found to be significantly higher in the group with vitamin B12 deficiency compared to the control group ( $p<0.001)$. There were no patients with a QTc value $>445$ ms in both the patient and control groups (Table 2).

In the patient group, the mean Tp-e interval was found to be $81.4 \pm 4.4 \mathrm{~ms}$. In the control group, the mean Tp-e interval was found to be $72.3 \pm 6.9 \mathrm{~ms}$. Tp-e interval was found to be statistically significantly higher in the patient group compared to the control group $(\mathrm{p}<0.001)$ (Table 3$)$.

In the patient group, the Tp-e/QT ratio and the Tp-e/ QTc ratio were found to be statistically significantly higher than the control group $(p<0.001$ and $p=0.001)$ (Table 3$)$.

QTd and QTcd values were found to be statistically significantly higher in the patient group compared to the control group $(p=0.009$ and $p<0.001)$ (Table 4). The minimum value of QTc in the patient group was found to be statistically significantly lower than the control group $(p<0.001)$ (Table 4).

No significant difference was found in both groups in terms of $E F$ and SF ( $p=0.408$ and $p=0.062)$. In the patient
Table 3. Comparison of Tp-e interval, Tp-e/QT ratio and Tp-e/QTc ratio in the patient and control groups

\begin{tabular}{|l|l|l|l|l|l|}
\hline & \multicolumn{2}{|l|}{ B12 group deficiency } & \multicolumn{2}{l|}{ Control group } & \\
\hline & Mean \pm SD & $\begin{array}{l}\text { Minimum } \\
\text { to } \\
\text { maximum }\end{array}$ & Mean \pm SD & $\begin{array}{l}\text { Minimum } \\
\text { to } \\
\text { maximum }\end{array}$ & p \\
\hline Tp-e & $81.4 \pm 4.4$ & $68-90$ & $72.3 \pm 6.9$ & $56-88$ & $<0.001^{\mathrm{a}}$ \\
\hline Tp-e/QT & $0.25 \pm 0.02$ & $0.19-0.33$ & $0.22 \pm 0.02$ & $0.17-0.27$ & $<0.001^{\mathrm{b}}$ \\
\hline $\begin{array}{l}\text { Tp-e/ } \\
\text { QTC }\end{array}$ & $0.21 \pm 0.01$ & $0.16-0.24$ & $0.18 \pm 0.02$ & $0.13-0.26$ & $<0.001^{\mathrm{c}}$ \\
\hline
\end{tabular}

aStudent's t-test, there is a significant difference between the groups in terms of Tp-e interval

'bstudent's t-test, there is a significant difference between the groups in terms of Tp-e interval/QT distance ratio

'Student's t-test, there is a significant difference between the groups in terms of Tp-e interval/ corrected QT distance ratio OTc: Corrected QT, SD: Standard deviation

group, IVSd and LVPWd (Left ventricular posterior wall diameter) values were found to be statistically significantly higher than the control group ( $p=0.013 ; p=0.040$ ).

A statistically significant negative correlation was found between vitamin B12 level and age in all cases included in the study ( $r=-0.175$ ) (Table 5). In all cases, a statistically significant negative correlation was found between vitamin B12 level and Tp-e interval, Tp-e/QT, Tp-e/QTc values ( $r=-$ $0.580 ; r=-0.511 ; r=-0.549$, respectively) (Table 5).

Heart rate was found to be statistically significantly lower in boys than in girls $(p=0.043)$. In terms of 
echocardiography measurements, LVESD (Left ventricular end-diastolic diameter) value was found to be statistically significantly higher in boys compared to girls $(p=0.042)$ (Table 6).

In the control group, heart rate was found to be statistically significantly lower in boys compared to girls $(p=0.034)$. There was no statistically significant difference in other ECG and ECO parameters ( $p>0.05)$.

\section{Discussion}

In our study, we found that in ECGs of children with isolated vitamin B12 deficiency, QT intervals and

\begin{tabular}{|c|c|c|c|c|c|}
\hline & \multicolumn{2}{|c|}{$\begin{array}{l}\text { Vitamin B12 deficiency } \\
\text { group }\end{array}$} & \multicolumn{2}{|c|}{ Control group } & \multirow[b]{2}{*}{$\mathbf{p}$} \\
\hline & Mean \pm SD & $\begin{array}{l}\text { Minimum } \\
\text { to } \\
\text { maximum }\end{array}$ & Mean \pm SD & $\begin{array}{l}\text { Minimum } \\
\text { to } \\
\text { maximum }\end{array}$ & \\
\hline \begin{tabular}{|l} 
QT \\
Minimum \\
\end{tabular} & $315.8 \pm 23.8$ & 261-384 & $321.6 \pm 27.3$ & $228-376$ & 0.204 \\
\hline \begin{tabular}{|l|} 
QT \\
Maximum \\
\end{tabular} & $340.9 \pm 25.5$ & $278-418$ & $344.0 \pm 24.4$ & $288-400$ & 0.476 \\
\hline QTd & $5.8 \pm 10.7$ & $7-4$ & 9 & 4-5 & $0.009^{a}$ \\
\hline \begin{tabular}{|l|} 
QTc \\
Minimum \\
\end{tabular} & $376.8 \pm 20.5$ & $326-423$ & $392.8 \pm 22.2$ & $341-454$ & $<0.001^{b}$ \\
\hline \begin{tabular}{|l|} 
QTc \\
Maximum \\
\end{tabular} & $413.1 \pm 19.1$ & $367-453$ & $419.2 \pm 17.9$ & $377-460$ & 0.077 \\
\hline QTcd & $36.1 \pm 13.0$ & $16-79$ & $27.4 \pm 14.7$ & $1-69$ & $<0.001^{c}$ \\
\hline \multicolumn{6}{|c|}{$\begin{array}{l}\text { aStudent's t-test, there is a significant difference between the groups in terms of } \\
\text { QT dispersion value } \\
\text { 'Student's t-test, there is a significant difference between the groups in terms of } \\
\text { corrected QT minimum value } \\
\text { 'Student's t-test, there is a significant difference between the groups in terms of } \\
\text { corrected QT dispersion value } \\
\text { OTc: Corrected QT, SD: Standard deviation }\end{array}$} \\
\hline
\end{tabular}

Table 5. Evaluation of vitamin B12 levels, age and electrocardiographic findings in all cases

\begin{tabular}{|l|l|l|}
\hline \multicolumn{2}{|l|}{} & B12 \\
\hline & r & p \\
\hline Age & -0.175 & $0.048^{\mathrm{a}}$ \\
\hline ECG & 0.103 & 0.246 \\
\hline Heart Rate & -0.580 & $<0.001^{\mathrm{b}}$ \\
\hline Tp-e & -0.511 & $<0.001^{\mathrm{c}}$ \\
\hline Tp-e/QT & -0.549 & $<0.001^{\mathrm{d}}$ \\
\hline Tp-e/QTC & &
\end{tabular}

apearson correlation, there is a significantly negative correlation between vitamin B12 and age.

bPearson correlation, there is a significantly negative correlation between vitamin B12 and Tp-e interval.

'Pearson correlation, there is a significantly negative correlation between vitamin B12 and Tp-e interval/QT distance ratio.

dPearson correlation, there is a significantly negative correlation between vitamin B12 and Tp-e interval/corrected QT distance ratio.

ECG: Electrocardiography, OTc: Corrected QT
Tp-E intervals, which can increase the predisposition to arrhythmia, were statistically significantly prolonged.

By affecting the activity of the sympathetic and parasympathetic system, vitamin B12 deficiency causes heart rate variability and cardiac autonomic dysfunction and also results in endothelial dysfunction and subclinical atherosclerosis with its role in cellular metabolic pathways inhibiting homocysteine metabolism, lipid peroxidation and free radical formation. There are studies where vitamin B12 deficiency is associated with left ventricular myocardial deformation $(1,2)$.

Herzlich et al. (9) found that left ventricular EF was significantly lower in the vitamin B12 deficient patients in the adult group. Kaya et al. (7) report that global and segmental myocardial deformation is impaired in patients with vitamin B12 deficiency and this disorder is associated with vitamin B12 levels.

The autonomic nervous system is the key regulator of the physiological functions of the cardiovascular system. It is known that vitamin B12 deficiency leads to the impairment of neuron myelinization and thus, causes cardiac autonomic dysfunction. In their study that was conducted with a group of patients with B12 deficiency, Beitzke et al. (10) evaluated the hemodynamic and autonomic responses of the patients with the tilt-table test. It was found that the baroreflex response was impaired in the vitamin B12 deficient group and a significant decrease was observed in the blood pressure measured after the tilt table test (10). In the paediatric patient group, the vitamin B12 deficiency was shown to cause the heart rate variability by affecting the activity of the sympathetic and parasympathetic system $(2,11)$. Öner et al. (12) compared low levels of vitamin B12 with the findings of postural orthostatic tachycardia syndrome (POTS) pattern in their study on adolescent children diagnosed with vasovagal syncope. It was reported that the deficiency may cause POTS with sympathetic system baroreceptor dysfunction in children with vitamin B12 deficiency (12).

Vitamin B12 has been associated with endothelial dysfunction and subclinical atherosclerosis with its role in cellular metabolic pathways that inhibit lipid peroxidation and free radical formation $(1,6)$. In their study on the paediatric group with vitamin B12 deficiency, Çelik et al. (1) report a significant relationship between carotid intimamedia thickness values measured in the early detection of atherosclerosis and the autonomic modulation of heart rate variability (HRV) (1).

Vitamin B12 deficiency is detected in various chronic diseases that develop with immune and inflammatory processes. Studies show that inflammation-related factors such as oxidative stress, nitric oxide production and exposure to various cytokines are associated with 
Table 6. Electrocardiographic and echocardiographic findings by gender in the vitamin B12 deficiency group

\begin{tabular}{|c|c|c|c|c|c|}
\hline & Male & & & Female & \\
\hline & Mean \pm SD & $\begin{array}{l}\text { Minimum to } \\
\text { Maximum }\end{array}$ & Mean \pm SD & $\begin{array}{l}\text { Minimum to } \\
\text { Maximum }\end{array}$ & $p$ \\
\hline \multicolumn{6}{|l|}{ ECG } \\
\hline Heart rate & $79.9 \pm 11.8$ & 56-107 & $87.4 \pm 16.5$ & 54-135 & $0.043^{a}$ \\
\hline QTc (msec) & $395 \pm 14.8$ & $366-430$ & $391.5 \pm 21.9$ & $335-429$ & 0.391 \\
\hline Tp-e & $82.4 \pm 4.5$ & $68-90$ & $80.5 \pm 4.3$ & $72-90$ & 0.084 \\
\hline Tp-e/QT & $0.25 \pm 0.02$ & $0.20-0.27$ & $0.25 \pm 0.02$ & $0.19-0.33$ & 0.320 \\
\hline Tp-e/QTC & $0.21 \pm 0.01$ & $0.16-0.24$ & $0.21 \pm 0.01$ & $0.17-0.23$ & 0.647 \\
\hline \multicolumn{6}{|l|}{ ECG } \\
\hline LVEDD (mm) & $43.0 \pm 4.9$ & $33-52$ & $40.6 \pm 5.0$ & $32-48$ & 0.057 \\
\hline $\operatorname{LVESD}(\mathrm{mm})$ & $25.7 \pm 6.5$ & $17-55$ & $23 \pm 3.1$ & 16-31 & $0.042^{b}$ \\
\hline $\mathrm{EF}(\%)$ & $74.4 \pm 5.6$ & $63-86$ & $73.7 \pm 7.3$ & 64-89 & 0.518 \\
\hline SF (\%) & $41.7 \pm 5.0$ & $34-53$ & $41.9 \pm 7.0$ & $34-60$ & 0.549 \\
\hline IVSd (mm) & $8.32 \pm 1.70$ & $6-11$ & $8.33 \pm 1.57$ & $5-11$ & 0.881 \\
\hline LVPWd $^{6}(\mathrm{~mm})$ & $8.32 \pm 1.64$ & $6-11$ & $8.33 \pm 1.85$ & $4-12$ & 0.908 \\
\hline \multicolumn{6}{|c|}{$\begin{array}{l}\text { LVEDD: Left ventricular end-diastolic diameter, LVESD: Left ventricular end-systolic diameter, EF: Ejection fraction, SF: Shortening fraction, IVSd: Interventricular septum } \\
\text { diameter, LVPWd: Left ventricular posterior wall diameter, ECG: Electrocardiography, SD: Standard deviation } \\
\text { aMann-Whitney U test, heart rate was found to be statistically significantly lower in boys than in girls }(p=0.043) \text {. } \\
\text { 'Mann-Whitney U test, left ventricular end-diastolic diameter value was found to be statistically significantly higher in boys compared to girls }\end{array}$} \\
\hline
\end{tabular}

increased intestinal permeability. Increased intestinal permeability is observed in another study conducted on patients with Behcet's syndrome and it is reported that the absorption of folate and vitamin B12 due to subclinical gastrointestinal inflammation may decrease (13). Vitamin B12 prevents oxidative stress with its role in cellular metabolic pathways that inhibit lipid peroxidation and free radical formation (14). In a study where the relationship between rheumatoid arthritis that develops with immune and inflammatory processes and vitamin B is examined, the detection of Th1-immune response and oxidative stress stimulated in parallel with decreased levels of vitamins B suggests that the immune activation may be accountable for homocysteine accumulation (15). Studies have been conducted in the childhood group to evaluate chronic diseases in which immune and inflammatory mechanisms play a role in the pathogenesis and their effects on the electrical activity of the heart. A significant relationship was found between Qt dispersion and Tp-e interval values in patients with Familial Mediterranean Fever, Kawasaki, hypothyroidism, celiac disease, ulcerative colitis and Crohn's disease (16-19).

In the study conducted by Isakov et al. (20) on 120 individuals between the ages of 40-70, one group was administered a multivitamin containing vitamin B12 and the other group was administered placebo for 8 weeks. The blood values and homocysteine levels showed that the cardiovascular risk parameters decreased in the group receiving vitamin supplements (20).
Several ECG parameters provide preliminary information on current or likely clinical situations. QT interval and T wave are significant as they indicate cardiac repolarization. It is shown that the increase in QT dispersion, which is considered to indicate regional heterogeneity in myocardial repolarization, causes serious ventricular arrhythmias and sudden cardiac death by way of re-entry (21). The Tp-e interval corresponds to the ventricular repolarization dispersion. Any increase in ventricular repolarization dispersion is regarded as an important risk factor for ventricular arrhythmias. The prolonged Tp-e interval represents the abnormal dispersion of ventricular repolarization and is associated with an increased risk of ventricular arrhythmia (14). Therefore, the Tp-e interval is a non-invasive screening method for arrhythmogenesis. Tp-e interval, the Tp-e/QT ratio and Tp-e/QTc ratio are parameters that represent the increase in the ventricular repolarization dispersion in recent studies. The prolonged Tp-e interval is associated with increased mortality in patients with long QT syndrome, Brugada syndrome and acute myocardial infarction. Since Tp-e/QT ratio is not affected by the heart rate and body weight, it is a more sensitive marker than others (4).

We could not find any study in the literature in English that evaluates the relationship between vitamin B12 and QT dispersion, which is used as an indicator of susceptibility to ventricular arrhythmia, and Tp-e interval in the adult or paediatric population. However, studies that evaluate these parameters with iron deficiency and vitamin $D$ in the paediatric population are available. Karadeniz et al. 
(22) report a significant negative correlation between the level of ferritin and Tp-e interval and QT dispersion values, which was thought to be associated with impaired cardiac hemodynamics due to iron deficiency anaemia. In their study conducted with 100 adolescent patients with the aim of investigating the relationship between vitamin $D$ and ventricular repolarization abnormalities, Bagrul et al. (23) reported that the effects of vitamin D on cardiac contractility and calcium haemostasis of the myocardium and QT dispersion and Tp-e interval values were found to be high. Tp-e interval and the Tp-Te/ QT ratio were found significantly increased in patients with ventricular tachycardia and ventricular fibrillation more than other in patients with ventricular premature complexes (VPC) and may be used as a novel non-invasive marker of differentiating malignant and benign VPC (24). In another article, Tp-e interval, Tp-e/QT ratio, and Tp-e/ QTc ratio, in patients with newly diagnosed COVID-19, were prolonged compared with normal healthy individuals (25). Our study suggests that both autonomic nervous system dysregulation and its function in cellular metabolic pathways and its endovascular damaging effects result in changes in the cardiac electrical activity.

In our study, QT dispersion and QTc dispersion values in the patient group were found to be statistically significantly higher compared to the control group $(p=0.009 ; p<0.001$, respectively). Tp-e interval was statistically significantly higher in the patient group compared to the control group $(p<0.001)$. In the patient group, the Tp-e/QT ratio and the Tp-e/QTc ratio were statistically significantly higher than in the control group ( $<<0.001 ; p<0.001$, respectively). In our study, no statistically significant difference was found in the heart rate in the patient group compared to the control group ( $p=0.118$ ). No significant difference was found between the patient and control groups in terms of P wave, PR interval, QRS complex and QT interval values.

In a study in which vitamin B12 and echocardiographic markers were evaluated in 367 patients who underwent angiography, a significantly decreased left ventricular EF was found in the B12 deficient group (9). In our study, LVEDD, LVESD, end-diastolic IVSd, end-diastolic LVPWd, EF and SF measurements were made in the patient and control groups as classical echocardiography measurements. No significant difference was not found in the EF and SF values in both groups. The mean IVSd and LVPWd values of the patient group were statistically significantly higher compared to the control group $(p=0.013$ and $p=0.040$, respectively).

A statistically significant negative correlation was found between the level of vitamin B12 and age in all cases included in the study $(r=-0.175)$. It is known that atherosclerosis resulting from endothelial dysfunction starts in childhood (6). The correlation between age and ECG parameters was evaluated in all cases. Any statistically significant correlation was not found between age and Tp-e interval, Tp-e/QT, Tp-e/QTc values.

\section{Study Limitations}

The sample size of the study is small. Our study is not planned for long term and thus, it does not provide the opportunity to evaluate the clinical findings of ECG changes in the future. Possible changes in the post-treatment ECG findings of the vitamin B12 deficient group could not be assessed due to time restrictions. However, our study is important for revealing the effect of isolated vitamin B12 deficiency on the electrical activity of the heart in order to eliminate the effect of other vitamin values on ECG findings, considering that iron deficiency and vitamin $D$ deficiency and ECG changes were examined in previous studies.

\section{Conclusion}

In conclusion, our study provides significant findings to detect early signs of cardiovascular events that may occur in the childhood age group with vitamin B12 deficiency. Repolarization differences compared to the normal population may reflect early subclinical findings associated with Vitamin B12 deficiency. Further prospective studies with larger samples are needed to identify these early period changes and their clinical significance.

\section{Authorship Contributions}

Concept: C.Y., N.S., Design: C.S., M.E., Data Collection or Processing: N.S., C.Y., Analysis or Interpretation: C.Y., K.S., M.E., Literature Search: C.Y., N.S., Writing: C.Y., K.S., M.E., N.S.

Conflict of Interest: No conflict of interest was declared by the authors.

Financial Disclosure: The authors declared that this study received no financial support.

\section{References}

1. Celik SF, Celik E. Subclinical atherosclerosis and impaired cardiac autonomic control in pediatric patients with Vitamin B12 deficiency. Niger J Clin Pract 2018;21:1012-6.

2. Sucharita S, Thomas T, Sowmya S, Krishnamachari S, Kurpad AV, Vaz M. Subclinical Vitamin B12 Deficiency and Heart Rate Variability Across Life Cycle. Curr Aging Sci 2016;9:217-23.

3. Castro-Torres Y, Carmona-Puerta R, Katholi RE. Ventricular repolarization markers for predicting malignant arrhythmias in clinical practice. World J Clin Cases 2015;3:705-20.

4. Gupta P, Patel C, Patel $H$, et al. T(p-e)/QT ratio as an index of arrhythmogenesis. J Electrocardiol 2008;41:567-74.

5. Sucharita S, Sowmya S, Thomas T, Kurpad AV, Vaz M. Plasma vitamin B12, methylmalonic acid and heart rate 
variability in healthy young Indian adults. Int J Vitam Nutr Res 2013;83:147-53.

6. Sadeghian S, Fallahi F, Salarifar M, et al. Homocysteine, vitamin B12 and folate levels in premature coronary artery disease. BMC Cardiovasc Disord 2006;6:38

7. Kaya Z, Bicer A, Yalcin F, et al. Impaired global and segmental myocardial deformation assessed by two-dimensional speckle tracking echocardiography in patients with vitamin B12 deficiency. Cardiol J 2014;21:60-6.

8. Guéant JL, Caillerez-Fofou M, Battaglia-Hsu S, et al. Molecular and cellular effects of vitamin B12 in brain, myocardium and liver through its role as co-factor of methionine synthase. Biochimie 2013;95:1033-40.

9. Herzlich BC, Lichstein E, Schulhoff $N$, et al. Relationship among homocyst(e)ine, vitamin B-12 and cardiac disease in the elderly: association between vitamin B-12 deficiency and decreased left ventricular ejection fraction. J Nutr 1996;126:1249-53.

10. Beitzke M, Pfister P, Fortin J, Skrabal F. Autonomic dysfunction and hemodynamics in vitamin B12 deficiency. Auton Neurosci 2002; 97:45-54.

11. Sucharita S, Dwarkanath $P$, Thomas T, Srinivasan $K$, Kurpad AV, Vaz M. Low maternal vitamin B12 status during pregnancy is associated with reduced heart rate variability indices in young children. Matern Child Nutr 2014;10:226-33.

12. Öner T, Guven B, Tavli V, Mese T, Yilmazer MM, Demirpence S. Postural orthostatic tachycardia syndrome (POTS) and vitamin B12 deficiency in adolescents. Pediatrics 2014;133:138-42.

13. Fresko I, Hamuryudan V, Demir M, et al. Intestinal permeability in Behçet's syndrome. Ann Rheum Dis 2001;60:65-6.

14. an de Lagemaat EE, de Groot LCPGM, van den Heuvel EGHM. Vitamin B12 in Relation to Oxidative Stress: A Systematic Review. Nutrients 2019;11:482.

15. Yxfeldt A, Wållberg-Jonsson S, Hultdin J, Rantapää-Dahlqvist S. Homocysteine in patients with rheumatoid arthritis in relation to inflammation and B-vitamin treatment. Scand J Rheumatol 2003;32:205-10.
16. Akın A, Unal E, Yıldırım R, Ture M, Balık H, Haspolat YK. Evaluation of QT dispersion and Tp-e interval in children with subclinical hypothyroidism. Pacing Clin Electrophysiol 2018;41:372-5.

17. Koca B, Kasapçopur O, Bakari S, et al. QT dispersion and cardiac involvement in children with Familial Mediterranean fever. Cardiol Young 2012;22:404-9.

18. Amoozgar H, Ahmadipour M, Amirhakimi A. QT Dispersion and T Wave Peak-to-end Interval Dispersion in Children with Kawasaki Disease. Int Cardiovasc Res J 2013;7:99-103.

19. Bornaun HA, Yılmaz N, Kutluk G, et al. Prolonged P-Wave and QT Dispersion in Children with Inflammatory Bowel Disease in Remission. Biomed Res Int 2017;2017:6960810.

20. Isakov VA, Bogdanova AA, Bessonov VV, et al. Effects of Multivitamin, Multimineral and Phytonutrient Supplementation on Nutrient Status and Biomarkers of Heart Health Risk in a Russian Population: A Randomized, Double Blind, Placebo Controlled Study. Nutrients 2018;10:120.

21. Pye MP, Cobbe SM. Mechanisms of ventricular arrhythmias in cardiac failure and hypertrophy. Cardiovasc Res 1992;26:74050 .

22. Karadeniz C, Özdemir R, Demirol M, et al. Low Iron Stores in Otherwise Healthy Children Affect Electrocardiographic Markers of Important Cardiac Events. Pediatr Cardiol 2017;38:909-14.

23. Bagrul D, Atik F. Association of vitamin D deficiency with ventricular repolarization abnormalities. Kardiol Pol 2019;77:853-8.

24. Zhao D, Liang B, Peng J, et al. Tp-e and (Tp-e)/QT ratio as a non-invasive risk factors for malignant ventricular arrhythmia in patients with idiopathic ventricular premature complexes. J Clin Lab Anal 2021;35:23636.

25. Yenerçağ $M$, Arslan $U$, Doğduş $M$, et al. Evaluation of electrocardiographic ventricular repolarization variables in patients with newly diagnosed COVID-19. J Electrocardiol 2020;62:5-9. 